\title{
Lungenfunktion im Alter: Brauchen wir neue Referenzwerte?
}

\section{Lung Function in the Elderly: Do We Need New Reference Values?}

Autoren

Institute
W. Marek¹, E. Marek', K. Mückenhoff², H. J. Smith ${ }^{3}$, N. Kotschy-Lang ${ }^{4}$, M. Kohlhäufl ${ }^{5}$

Institut für Arbeitsphysiologie an der Augusta-Kranken-Anstalt, Bochum

2 Institut für Physiologie, Ruhr-Universität Bochum

Viasys Health Care, Höchberg

${ }^{4}$ Berufsgenossenschaftliche Klinik für Berufskrankheiten Falkenstein

Klinik Schillerhöhe, Zentrum für Pneumologie und Thoraxchirurgie, Stuttgart-Gerlingen eingereicht 27.8.2008 akzeptiert nach Revision 31. 1.2009

\section{Bibliografie}

DOI $10.1055 / \mathrm{s}-0028-1119704$

Pneumologie 2009; 63:

235-243 @ Georg Thieme

Verlag KG Stuttgart · New York ISSN 0934-8387

\section{Korrespondenzadresse \\ Dr. rer. nat. PD \\ Wolfgang Marek}

Institut für Arbeitsphysiologie an der Augusta-Kranken-Anstalt Bergstr. 23

44791 Bochum

Wolfgang.Marek@rub.de

\section{Zusammenfassung \\ $\nabla$}

Einleitung: Die Bevölkerungsstruktur in Europa und die anthropometrischen Daten der Bewohner haben sich in den letzten drei Jahrzehnten deutlich verändert. Dieses wirft erneut die Frage nach der Verwendbarkeit der weit verbreiteten Referenzwerte der Europäischen Gemeinschaft für Kohle und Stahl (EGKS), die sich auf die ventilatorische Lungenfunktion beziehen, auf. Die EGKSReferenzwerte wurden Ende der 70er-Jahre vornehmlich an Probanden im mittleren Lebensabschnitt gewonnen, für ältere Probanden muss daher eine Extrapolation über den Geltungsbereich der Sollwertfunktionen hinaus durchgeführt werden, die insbesondere unter dem Aspekt der Begutachtung zu Unsicherheiten und zu Nachteilen in der Beurteilung führt. Die EGKS-Werte, insbesondere die unteren Grenzwerte, werden im Vergleich zu aktuellen Untersuchungen [1,2] vielfach als zu niedrig angesehen und sollten daher möglichst bald ersetzt werden.

Methoden: Wir überprüften die statischen und dynamischen Lungenvolumina und die maximalen Atemstromstärken an einem Kollektiv von 176 anamnestisch lungengesunden, nie rauchenden Männern im Alter von 20 - 90 Jahren und bezogen die Ergebnisse auf die Referenzwerte der EGKS, der SAPALDIA und LuftiBus.

Ergebnisse: Der Trend in der Altersabhängigkeit spirometrischer Parameter (VC, FVC, $\mathrm{FEV}_{1}, \mathrm{FEV}_{1} \%$ FVC, PEF, $\mathrm{MEF}_{75,50,25}$ ) kann über den Altersbereich von 70 Jahren hinaus mit einer linearen Funktion $(\mathrm{y}=-\mathrm{m} \times$ Alter $+\mathrm{n})$ beschrieben werden. Für die Einsekundenkapazität $\left(\mathrm{FEV}_{1}\right)$ ergab sich die Funktion $\mathrm{FEV}_{1}=-0,046 \times$ Alter $+6,11 ; \mathrm{r}=0,88$. Diese lag bei den jüngeren Männern bei $108 \pm 9,9 \%$, bei den mittelalten bei $105 \pm 13,7 \%$ und bei den älteren Probanden bei 97,3 $\pm 12,4 \%$ der EGKS-Referenzwerte. Bei allen gemessenen Parametern wurde eine gegenüber den EGKS-Werten größere Steigung der Funktionen in Abhängigkeit vom Al-

\section{Abstract \\ $\nabla$}

Introduction: The structure of our aging population has significantly changed in the last three decades as have also the anthropometric data. Therefore, the question arises as to whether or not the largely accepted reference values for ventilatory lung function, which were suggested by the European Community for Coal and Steel (EGKS), may still be used today, since these values were obtained in the 1960s from subjects in a limited age range. For the elderly, the measured values are deduced by extrapolation beyond the range of reference equations which had been obtained in a different population. Therefore decisions concerning elderly and smaller subjects concerning remuneration due to impaired lung function after industrial exposure on the basis of EGKS values are questionable.

Methods: We have examined lung function using pneumotachography for recording static lung volumes and flow-volume curves in 176 asymptomatic non-smoking males, aged 20 to 90 years, and correlated the results to the reference values of the EGKS, SAPALDIA and LuftiBus.

Results: The age dependence of respiratory parameters (VC, FVC, $\mathrm{FEV}_{1}, \mathrm{FEV}_{1} \% \mathrm{FVC}, \mathrm{PEF}$, $\mathrm{MEF}_{75,50,25}$ ) for the healthy subjects can be described with a linear function $(y=-m \times$ age $+n)$. The forced expiratory volume in one second, $\mathrm{FEV}_{1}$, is calculated by $\mathrm{FEV}_{1}=-0.046 \times$ age +6.11 ; $r=0.88$. Mean FEV1 for younger subjects was found to be $108 \pm 9.9 \%$ of the EGKS reference values, $105 \pm 13.7 \%$ in the middle-aged group and $97.3 \pm 12.4 \%$ in the older subjects. All measured parameters concerning lung function can be described as linear functions of age which are steeper than those described by the EGKS reference values. The steeper slope in age dependency was also seen in other investigated parameters. The correlation of lung function parameters to height largely follows the EGKS predictions. 
ter gefunden. Die Korrelation der Parameter zur Körpergröße entspricht hingegen weitgehend den EGKS-Werten.

Schlussfolgerungen: Die Messwerte der ventilatorischen Lungenfunktion von lungengesunden jüngeren Probanden liegen über den Sollwerten der EGKS, während die der älteren Probanden im Mittel leicht unter den extrapolierten Sollwerten der EGKS liegen. Der Altersgang verläuft demnach steiler als durch die EGKS-Referenzwerte beschrieben. Unsere Ergebnisse erlauben durchaus eine Extrapolation der Sollwerte über den angegebenen Bereich hinaus. Das Problem liegt vornehmlich in der Beurteilung anhand des unteren Grenzwertes für kleinere und ältere Menschen. Die alternativ diskutierten Referenzwertgleichungen der SAPALDIA-Studie, der NHANES- und zum Teil auch der LuftiBus-Studie liegen zwar höher, doch können sie den benötigten Umfang an Messgrößen oder den erforderlichen Altersbereich nicht abdecken. Eine multizentrische Studie zur Gewinnung neuer Sollwerte wäre daher notwendig, um die Probleme zu lösen.

\section{Einleitung}

Die Veränderungen in der Bevölkerungsstruktur (z. B. steigender Anteil der > 65-Jährigen an der Gesamtbevölkerung) und den anthropometrischen Daten (z.B. zunehmende Körpergröße junger Erwachsener), die in den letzten drei Jahrzehnten registriert wurden, verstärken die Bedenken über die Verwendbarkeit der Referenzwerte für die ventilatorische Lungenfunktion der EGKS von Quanjer und Mitarbeitern [3,4]. Die verbreiteten Werte der EGKS, auf die sich auch die aktuellen Empfehlungen der Deutschen Atemwegsliga zur Einstufung von Lungenfunktionsstörungen stützen [5], wurden aus Formulierungen vorhandener Referenzwertangaben gewonnen und stammten von Probanden in einem begrenzten Alters- und Größenbereich. Die EGKS-Referenzwerte werden in der Literatur unter verschiedenen Aspekten kritisiert. Argumente für und gegen neue Referenzwerte sind in - Tab. 1 exemplarisch zusammengestellt.

Die Empfehlungen der EGKS basieren auf einer Zusammenführung damals bekannter Referenzgleichungen für die „Normalwerte“ der Lungenfunktion und ihrer Grenzwerte. Als Untergrenze oder 5\% Perzentile wird das 1,64-fache der Standardabweichung der Residuen (RSD) vom Sollwert abgezogen. Die EGKS-Referenzwerte wurden für ein Lebensalter bis 70 Jahren beschrieben, wobei die Altersklasse über 65 Jahren bereits unterrepräsentiert war. Heute sind knapp 16,7 Mio. Bundesbürger (20\%) älter als 65 Jahre. In 30 Jahren werden bereits 23,5 Mio. Bundesbürger oder $32 \%$ mehr als 65 Jahre alt sein (DE-STATIS). Das heißt, diese Gruppe wird doppelt so groß sein, wie die der jungen Leute unter 19 Jahren.

Für ältere Probanden/Patienten muss schon heute eine Extrapolation über die Grenzen der gültigen Lungenfunktions-Werte hinaus durchgeführt werden, was in der Vergangenheit zu kontroversen Diskussionen geführt hat und nicht generell empfohlen wurde. Darüber hinaus sind die EGKS-Referenzwerte und insbesondere die unteren Grenzwertangaben sehr niedrig im Vergleich zu den aktuellen Querschnittsuntersuchungen wie im Rahmen der SALPALDIA [6,7] oder der NHANES III [1] ermittelt wurden. Ältere und kleine Menschen werden erheblich benachteiligt, wenn Einstufungen nach den EGKS-(Grenz-)Werten durchgeführt werden. So sind der Referenzwert und die Solluntergrenze für die FVC nach SAPALDIA im Mittel um 9\% höher als die der EGKS. Jedoch sind die Unterschiede im hohen Maße al-
Conclusions: Measured lung function values of healthy younger and elderly subjects showed a close correlation to the extrapolated reference values of the EGKS. Our results relating to normal lung function justify an extrapolation of the reference equations beyond the common ranges of age while applying the same limitations as described for subjects in the middle-age range. Our results permit an extrapolation of EGKS values beyond the range of the reference values and can be used for the classification of impaired lung function in older subjects. The alternatively discussed reference equations of the SALPADIA Study, of NHANES and partially of the LuftiBus Study are higher, but do not cover all the necessary parameters and/or age ranges. A multicentric study for contemporary reference values should be performed in order to solve the problems concerning valid reference values.

tersabhängig. Bei jungen und großen Männern beträgt die Differenz lediglich $2 \%$, bei älteren kleinen Männern erhöht sie sich jedoch auf bis zu 32\%. Dieser Trend findet sich mehr oder weniger stark ausgebildet bei allen dynamischen Lungenfunktionsparametern und gilt sowohl für Männer als auch für Frauen. Kleinere und ältere Menschen müssen somit erhebliche Verluste ihrer Lungenfunktion erreichen, um als „krank“ eingestuft zu werden.

Tab. 1 Brauchen wir neue Referenzwerte für die Lungenfunktion?

\begin{tabular}{|c|c|}
\hline Contra! & Pro? \\
\hline $\begin{array}{l}\text { Referenzwerte der EGKS sind } \\
\text { seit } 25 \text { Jahren flächendeckend } \\
\text { etabliert. }\end{array}$ & $\begin{array}{l}\text { Anthropometrische Daten haben } \\
\text { sich zwischenzeitlich verändert. }\end{array}$ \\
\hline $\begin{array}{l}\text { Einfache und überschaubare } \\
\text { lineare Funktionen, berücksich- } \\
\text { tigen Alter und Körpergröße. }\end{array}$ & $\begin{array}{l}\text { Messtechnik, Datengewinnung, } \\
\text {-verarbeitung und die graphische } \\
\text { Präsentation sind inzwischen } \\
\text { deutlich verbessert. }\end{array}$ \\
\hline $\begin{array}{l}\text { Einfache Lösung zur Erweiterung } \\
\text { des Altersbereichs: lineare Extra- } \\
\text { polation über das } 65 \text {. Lebensjahr } \\
\text { hinaus. }\end{array}$ & $\begin{array}{l}\text { Menschen werden erheblich älter, } \\
\text { der Anteil der über 65-Jährigen } \\
\text { steigt innerhalb der nächsten } \\
\text { Generation auf über } 25 \% \text { der } \\
\text { Bevölkerung an. }\end{array}$ \\
\hline $\begin{array}{l}\text { Multizentrisches Forschungs- } \\
\text { projekt, } 20000 \text { Probanden oder } \\
\text { mehr, gleiche Geräte, Personal, } \\
\text { Schulung, Koordination, doch nur } \\
\text { ein Querschnitt. }\end{array}$ & $\begin{array}{l}\text { EGKS-Sollwerte und die Grenz- } \\
\text { werte sind zu niedrig, insbeson- } \\
\text { dere für ältere und kleinere Per- } \\
\text { sonen. }\end{array}$ \\
\hline $\begin{array}{l}\text { EGKS-Referenzwerte sind als } \\
\text { einzige komplett, sie enthalten } \\
\text { statische und dynamische } \\
\text { Lungenfunktionsparameter, ein- } \\
\text { schließlich des Residualvolumens. }\end{array}$ & $\begin{array}{l}\text { Ein fester Grenzwert als Sollwert } \\
-1,64 \times \text { RSD benachteiligt ins- } \\
\text { besondere kleinere und ältere } \\
\text { Personen erheblich. }\end{array}$ \\
\hline $\begin{array}{l}\text { Definieren wir als unteren Grenz- } \\
\text { wert einen festen \%-Satz vom Soll- } \\
\text { mittelwert, dann werden alte und } \\
\text { kleine Patienten weniger benach- } \\
\text { teiligt, vgl. Deutsche Atemwegs- } \\
\text { liga. }\end{array}$ & $\begin{array}{l}\text { Ein fester \%-Satz vom Mittelwert } \\
\text { ist wissenschaftlich nicht begrün- } \\
\text { det. Es ist unbekannt, ob die } \\
\text { Streuung mit Abnahme der Soll- } \\
\text { werte kleiner wird oder nicht. }\end{array}$ \\
\hline $\begin{array}{l}\text { Wer sollte Interesse haben, ein } \\
\text { Sollwerte-Projekt mit einem } \\
\text { Finanzvolumen von einigen } \\
\text { Millionen Euro zu finanzieren? } \\
\text { Wer würde davon letztlich } \\
\text { profitieren? }\end{array}$ & $\begin{array}{l}\text { Ärzte brauchen verlässliche An- } \\
\text { gaben für die Diagnostik und für } \\
\text { Gutachten gegenüber Patienten, } \\
\text { Versicherungen und Berufsge- } \\
\text { nossenschaften. }\end{array}$ \\
\hline
\end{tabular}


Diese ungleiche Behandlung ist weder ethisch noch wissenschaftlich vertretbar.

Auch die Empfehlungen der „ATS/ERS Task Force: lung function testing“ konnten das Problem des eingeschränkten Gültigkeitsbereichs der Referenzwerte und der starren Festlegung der unteren Grenzwerte nicht lösen [8].

Die Diskussion um die Referenzwerte der EGKS und ihren möglichen Ersatz durch die Formulierungen der SAPALDIA-Studie wird seit mehr als 10 Jahren geführt [9-11]. Aktuell erschien dazu ein Leserbrief von Merget und Degens im ERJ [12], in dem die Abkehr von den EGKS-Sollwerten und die sofortige Übernahme der Referenzgleichungen der SAPALDIA-Studie gefordert wurde. In ihrer Antwort sind die Vertreter der ATS/ERS Task Force der Meinung, dass die SAPALDIA-Werte vielleicht nützlich für Schweizer Lungenfunktionslabore seien und fordern nachdrücklich eine europaweite Sollwertstudie, die neben den statischen und dynamischen Lungenfunktionsparametern auch die Diffusionseigenschaften der Lunge einschließen sollen [12].

Drei Modelle für den Altersgang der Lungenfunktionsparameter nach dem 65. Lebensjahr lassen sich ableiten: ein „normaler“ Altersgang erlaubt eine lineare Extrapolation über das 65. Lebensjahr hinaus. Ältere Lungengesunde könnten konstante Lungenfunktionswerte beibehalten. Durch Akkumulation von Erkrankungen könnten Ältere eine schnellere Abnahme der Lungenfunktion aufweisen, wie für Patienten mit COPD oder starken Rauchern bereits gezeigt werden konnte [13-15].

Zumindest für einen Interimszeitraum ist ein pragmatisches Vorgehen zur Lösung des Sollwertproblems dringend notwendig. Eine lineare Extrapolation der EGKS-Sollwerte wäre die einfachste Lösung. Gerätetechnisch ist die Extrapolation von den meisten Geräteherstellern eingerichtet und wird stillschweigend bereits breitflächig praktiziert. Dieses Vorgehen entbehrt jedoch der wissenschaftlichen Grundlage und es stellt sich die Frage, ob man sich im Falle einer als normal eingestuften Lungenfunktion auf die extrapolierten Werte verlassen kann.

Wir überprüften daher an einem kleinen, jedoch sehr sorgfältig ausgesuchten Referenzkollektiv gesunder männlicher Nichtraucher im Lebensalter zwischen 20 und 90 Jahren, ob eine Extrapolation der EGKS-Referenzwerte zu vertreten ist, oder ob im höheren Lebensalter die Abnahme der Lungenfunktion einen anderen, nicht linearen zeitlichen Verlauf nimmt.

Die aktuellen Referenzwertangaben aus der Literatur sind unvollständig bezüglich der Parameterauswahl (Hankinson [1], nur $\mathrm{FVC}, \mathrm{FEV}_{1}, \mathrm{FEV}_{6}, \mathrm{PEF}, \mathrm{FEF}_{25-75}$ ) des Altersbereichs (Brändli [6] 20-60 Jahre, FEV ${ }_{1}$, FVC, FEV 1 \%FC, PEF, $\mathrm{MEF}_{75,50,25}, \mathrm{MEF}_{25-75}$ ), oder enthalten, wie die LuftiBus-Studie, einen erheblichen Selektionsbias in dem nur Probanden untersucht worden sind, die gegen Bezahlung eine Lungenfunktionsprüfung durchführen ließen (Kuster [2] FEV 1 , FVC, FEV $\%$ FVC, PEF, $\mathrm{MEF}_{75,50,25}$ ). Für eine umfassende Lungenfunktionsdiagnostik werden die statischen und dynamischen Lungenvolumina aus der Spirometrie sowie das Residualvolumen (RV) benötigt, dazu der PEF und die $\mathrm{MEF}_{75,50,25}{ }^{-}$ Werte aus der Fluss-Volumen-Kurve. Komplett liegen die Parameter ausschließlich für die EGKS-Sollwerte vor.

\section{Material und Methoden \\ $\nabla$}

Referenzwerte und untere Grenzwerte

Anhand der FEV 1 soll der Stellenwert der Referenzwerte exemplarisch erläutert werden. Die EGKS-Sollwertformel beschreibt einen linearen Zusammenhang der $\mathrm{FEV}_{1}$ mit der Körpergröße (H in $\mathrm{m}$ ) und dem Lebensalter ( $\mathrm{A}$ in Jahren): $\mathrm{FEV}_{1}=4,30 \times \mathrm{H}-$ $0,029 \times A-2,49$. Das bedeutet: die $\mathrm{FEV}_{1}$ nimmt mit jedem $\mathrm{cm}$ Körpergröße um $43 \mathrm{~mL}$ zu und mit jedem weiteren Lebensjahr um $29 \mathrm{~mL}$ ab. Zwischen 25-jährigen $200 \mathrm{~cm}$ großen und $160 \mathrm{~cm}$ großen jungen Männern berechnet man eine Differenz der $\mathrm{FEV}_{1}$ von 5,5 $\mathrm{L}$ zu 2,2 L, = 3,3 $\mathrm{L}$ entsprechend $60 \%$. Vergleichbar sind die Referenzwertformulierungen für Frauen: $\mathrm{FEV}_{1}=3,95 \times \mathrm{H}-$ $0,0259 \times A-2,60$. Das bedeutet: die $\mathrm{FEV}_{1}$ nimmt bei den Frauen mit jedem cm Körpergröße um $40 \mathrm{~mL}$ zu und mit jedem weiteren Lebensjahr um $26 \mathrm{~mL}$ ab.

Der untere Grenzwert nach EGKS ist festgelegt durch den Abzug von 1,64 × RSD, der Standardabweichung der Residuen vom Sollwert, also unabhängig vom Lebensalter und der Körpergröße werden vom Referenzwert des $\mathrm{FEV}_{1}$ jeweils $0,84 \mathrm{~L}$ abgezogen, um die $5 \%$ Perzentile zu erreichen. Für ältere und kleinere Männer ist dieser Grenzwert unverhältnismäßig tief angelegt und erreicht deutlich pathologische Werte, sodass z.B. die Prognosen von lungenchirurgischen Eingriffen falsch eingeschätzt werden und Tumorresektionen nicht mehr durchgeführt werden.

Die $5 \%$ Perzentile wird von $200 \mathrm{~cm}$ großen 25-Jährigen erreicht, wenn $17 \%$ vom Sollwert abgezogen werden, bei einem 90 -jährigen $200 \mathrm{~cm}$ großen Mann entspricht dies $21 \%$. Bei einem $160 \mathrm{~cm}$ großen Mann sind dies mit 25 Jahren bereits $23 \%$, jedoch mit 90 Jahren sogar $51 \%$, also nahezu die Hälfte des Referenzwertes. Trägt man die Differenz der 5er-Perzentilen für unterschiedlich große Männer über dem Lebensalter auf, so ergeben sich mit abnehmender Körpergröße Funktionen, die mit zunehmendem Lebensalter steiler verlaufen.

Die Graduierung obstruktiver Atemwegserkrankungen durch die Deutsche Atemwegsliga führte zu akzeptableren Werten, da nicht ein Absolutbetrag vom jeweiligen Sollwert abgezogen wird, sondern ein fester Prozentsatz. Die Stärke der Obstruktion wurde in fünf Stufen (leicht, mäßig, mittelschwer, schwer, sehr schwer) eingeteilt, entsprechend $<70 \%,<60 \%,<50 \%,<35 \%$ des Sollwertes.

\section{Anthropometrische Daten, Probandenauswahl}

An 176 niemals rauchenden Männern im Alter von 20-90 Jahren, aufgeteilt in drei Altersgruppen (20 - 40 Jahre, 41 - 65 Jahre und 66-90 Jahre), die anamnestisch keine Lungen-, Herz- oder weitere Organbeschwerden angaben, überprüften wir die Messwerte für die ventilatorische Lungenfunktion. Die Untersuchungen erfolgten zwischen Januar 2000 und Dezember 2002 in der Klinik für Pneumologie der Augusta-Kranken-Anstalt Bochum (Meereshöhe $+130 \mathrm{~m}$ ).

Die Probanden, sämtlich Mitteleuropäer, rekrutierten sich aus einem Kollektiv lungengesunder Nichtraucher, aus nichtrauchenden Mitarbeitern der Klinik und Freiwilligen, aus Probanden, die sich zu einem Gesundheitstest aus versicherungstechnischen oder persönlichen Gründen vorstellten, bzw. aus Patienten, die in Vorbereitung auf einen minimal-chirurgischen Eingriff lungenfunktionsanalytisch untersucht wurden. Unter den Probanden waren keine Leistungssportler und keine Arbeitnehmer, die körperlich besonders beansprucht wurden. Nach einer ausführlichen Anamnese, mit Schwerpunkten auf pulmonale, kardio-vaskuläre und andere Erkrankungen, fragten wir nach dem Rauch- 
Tab.2 Anthropometrische Daten der in die Studie aufgenommenen jüngeren, mittelalten und älteren lungengesunden Probanden.

\begin{tabular}{|c|c|c|c|c|c|c|c|c|c|c|c|c|}
\hline & \multicolumn{4}{|c|}{ Ältere Probanden ( $n=53$ ) } & \multicolumn{4}{|c|}{ Mittelalte Probanden $(n=50)$} & \multicolumn{4}{|c|}{ Junge Probanden $(n=73$ ) } \\
\hline & $\begin{array}{l}\text { Alter } \\
\text { (Jahre) }\end{array}$ & $\begin{array}{l}\text { Größe } \\
(\mathrm{cm})\end{array}$ & $\begin{array}{l}\text { Gewicht } \\
\text { (kg) }\end{array}$ & BMI & $\begin{array}{l}\text { Alter } \\
\text { (Jahre) }\end{array}$ & $\begin{array}{l}\text { Größe } \\
(\mathrm{cm})\end{array}$ & $\begin{array}{l}\text { Gewicht } \\
\text { (kg) }\end{array}$ & BMI & $\begin{array}{l}\text { Alter } \\
\text { (Jahre) }\end{array}$ & $\begin{array}{l}\text { Größe } \\
(\mathrm{cm})\end{array}$ & $\begin{array}{l}\text { Gewicht } \\
\text { (kg) }\end{array}$ & BMI \\
\hline MW & 74,0 & 172 & 77,2 & 25,9 & 56,8 & 176 & 83 & 26,7 & 27,3 & 182 & 77,1 & 23,8 \\
\hline STD & $\pm 6,4$ & $\pm 7,3$ & $\pm 10,4$ & $\pm 2,4$ & $\pm 7,9$ & $\pm 5,8$ & $\pm 8,2$ & $\pm 2,2$ & $\pm 5,0$ & $\pm 6,7$ & $\pm 10,1$ & $\pm 2,7$ \\
\hline MAX & 90 & 186 & 100 & 31,6 & 65 & 190 & 106 & 32,1 & 40 & 198 & 115 & 33,3 \\
\hline MIN & 66 & 159 & 60 & 21,7 & 41 & 167 & 68 & 20,5 & 21 & 164 & 57 & 18,8 \\
\hline MED & 73 & 173 & 75 & 25,8 & 58 & 175 & 80 & 26,6 & 26 & 180 & 75 & 33,3 \\
\hline
\end{tabular}

\begin{tabular}{|c|c|c|c|c|}
\hline Messgröße & $\begin{array}{l}\text { Ist-Werte, } \\
\text { lin. Regression }\end{array}$ & $\mathrm{R}^{2}$ & $\begin{array}{l}\text { Lin. Regression } \\
\text { Ist-Werte in \%SolI }\end{array}$ & $\mathbf{R}^{2}$ \\
\hline VCIN (I) & $y=-0,046 x+7,01$ & $0,70^{* *}$ & $y=-0,174 x+108$ & $0,10^{* *}$ \\
\hline FVC (I) & $y=-0,046 x+7,03$ & $0,74^{* *}$ & $y=-0,220 x+114$ & $0,11^{* *}$ \\
\hline FEV1 (1/min) & $y=-0,046 x+6,11$ & $0,78^{* *}$ & $y=-0,218 x+115$ & $0,13^{* *}$ \\
\hline FEV1\%VCIN & $y=-0,185 x+90,0$ & $0,52^{* *}$ & $y=0,011 x+101$ & $0,01 \mathrm{n} . \mathrm{s}$ \\
\hline PEF (1/min) & $y=-0,078 x+13,2$ & $0,53^{* *}$ & $y=-0,284 x+119$ & $0,12^{* *}$ \\
\hline MEF75 (I/min) & $y=-0,045 x+10,3$ & $0,09^{* *}$ & $y=0,024 x+106$ & 0,01 n.s. \\
\hline MEF50 (I/min) & $y=-0,049 x+7,32$ & $0,46^{* *}$ & $y=-0,238 x+113$ & $0,04^{*}$ \\
\hline MEF25 (I/min) & $y=-0,047 x+3,88$ & $0,63^{* *}$ & $y=-0,821 x+120$ & $0,10^{* *}$ \\
\hline
\end{tabular}

Tab. 3 Altersabhängigkeit der Lungenfunktionsparameter lungengesunder männlicher Probanden $(\mathrm{N}=164)$.

n. s. nicht signifikant; ${ }^{*} \alpha<0,05 ;{ }^{* *} \alpha<0,01$; \# Lineare Regression der Ist-Werte angegeben als \%Soll der EGKS.

verhalten, nach Allergien und beruflichen bzw. freizeitlichen Schadstoffexpositionen und nach Medikamenten. Die anthropometrischen Daten sind in $\bullet$ Tab. 2 zusammengestellt.

\section{Lungenfunktionsuntersuchungen}

Die Lungenfunktionsprüfung erfolgte gemäß den Empfehlungen der Europäischen Gemeinschaft für Kohle und Stahl (EGKS)[16]. Für die Spirometrie und Fluss-Volumen-Kurve wurden die Sollwerte der EGKS zu Grunde gelegt. In einem volumenkonstanten Ganzkörperplethysmographen (MasterLab, Jaeger-Tönnies, Würzburg) führten wir bei geöffneter Tür eine Spirometrie mit dreimaliger Messung der inspiratorischen Vitalkapazität $\left(\mathrm{VC}_{\mathrm{IN}}\right)$ durch $[17,18]$. Im zweiten Schritt erfolgte die Ermittlung der Fluss-Volumen-Kurve. Aus der endexspiratorischen Atemlage wurde zunächst maximal bis zum Residualvolumen (RV) ausgeatmet, dann folgten drei forcierte maximale Atemmanöver.

\section{Datenanalyse}

Die Ergebnisse werden als Mittelwerte und Standardabweichungen $\left(x \pm s_{x}\right)$, sowie als Median angegeben. Unter Verwendung von Fisher's paarigem T-Test wurde geprüft, ob die Mittelwerte der gemessenen Variablen signifikant von den Sollwertangaben der EGKS bzw. von den SAPALDIA- oder den LuftiBus-Empfehlungen abwichen [19]. Ein p-Wert kleiner als 0,05 wurde als signifikant angenommen. Lineare Korrelationsgleichungen wurden für den Altersbereich zwischen 20 und 90 Jahren und für Körpergrößen zwischen 160 und $200 \mathrm{~cm}$ berechnet. Die Messwerte wurden in Bezug auf die Referenzwerte für die Alters- und Größenabhängigkeit berechnet und auf ihre Signifikanz überprüft.

\section{Ergebnisse \\ $\nabla$}

Anthropometrische Daten

Die 176 Probanden wurden in 3 Altersgruppen (20-40, 41-65 und 66 - 90 Jahre) eingeteilt und miteinander verglichen. Der Altersmedian der jüngeren Männer lag bei 26 Jahren, der der älteren Männer bei 73 Jahren, $6 \%$ waren älter als 80 Jahre.

Die Auswertung der Querschnittserhebung ergab eine signifikante $(r=0,52, \alpha<0,01)$ Beziehung zwischen dem Lebensalter und der Körpergröße (Größe = - 0,183 $\times$ Alter + 186). Die Körpergröße war pro Dekade Lebensalter im Mittel um 1,83 cm geringer. Die mittlere Körpergröße eines 25-jährigen Probanden betrug $182 \mathrm{~cm}$, die eines 80-jährigen hingegen $171 \mathrm{~cm}$.

Der BMI zeigte eine signifikante Zunahme mit dem Lebensalter $(\mathrm{BMI}=0,049 \times$ Alter $+22,6, r=0,37, \alpha<0,01)$. Für die jüngeren Männer bestimmten wir einen mittleren BMI von 23,8 $\pm 2,7$ und für die älteren Probanden einen signifikant höheren Wert von $25,9 \pm 2,4(\mathrm{p}<0,005)$. Der BMI nahm tendenziell mit steigender Körpergröße ab (BMI $=-0,0723 \times$ Größe $+37,9, r=0,19, \alpha>0,05)$.

\section{Lungenfunktionsprüfungen}

Die $\mathrm{VC}_{\mathrm{IN}}$ als statisches Lungenvolumen, die forcierten Volumina FVC und $\mathrm{FEV}_{1}$, sowie die maximalen exspiratorischen Stromstärken PEF und $\mathrm{MEF}_{75,50,25}$ der Probanden zeigten eine signifikante lineare Beziehung zum Lebensalter $(\alpha<0,01)$, die über den Gültigkeitsbereich der EGKS-Referenzwertfunktionen hinausgeht ( $\bullet$ Tab. 3, links). Die Messwerte, berechnet als \% der jeweiligen Referenzwerte, zeigen ebenfalls eine signifikante Korrelation zum Lebensalter. Das Ergebnis zeigt, dass die Altersabhängigkeiten der EGKS-Werte im Vergleich zu den hier untersuchten Probanden zu flach angelegt sind ( $\bullet$ Tab. 3 , rechts).

Die Messwerte der Lungenfunktion zeigen hingegen die von den EGKS Referenzwerten vorausgesagte (lineare) Korrelation zur Körpergröße ( $\bullet$ Tab.4, links). Für die Messwerte in \% der Referenzwerte besteht keine Korrelation zur Körpergröße, d. h. die Beziehung zur Körpergröße wird von EGKS-Referenzwerten zutreffend repräsentiert ( $\bullet$ Tab. 4 , rechts). 


\begin{tabular}{|c|c|c|c|c|}
\hline Messgröße & $\begin{array}{l}\text { Ist-Werte, } \\
\text { lin. Regression }\end{array}$ & $\mathrm{R}^{2}$ & $\begin{array}{l}\text { Lin. Regression } \\
\text { Ist-Werte in \%Soll\# }\end{array}$ & $R^{2}$ \\
\hline VCIN (I) & $y=0,104 x-13,6$ & $0,46^{* *}$ & $y=0,083 x+84,4$ & 0,003 n.s. \\
\hline FVC (I) & $y=0,103 x-13,5$ & $0,46^{* *}$ & $y=0,131 x+79,8$ & 0,007 n.s. \\
\hline FEV1 (1/min) & $y=0,091 x-12,2$ & $0,41^{* *}$ & $y=0,220 x+65,0$ & 0,016 n.s. \\
\hline FEV1\%VCIN & $y=0,267 x+33,9$ & $0,08^{* *}$ & $y=-0,001 x+103$ & 0,001 n.s. \\
\hline PEF (I/min) & $y=0,163 x-19,5$ & $0,30^{* *}$ & $y=0,478 x+20,7$ & $0,043^{*}$ \\
\hline MEF75 (I/min) & $y=0,066 x-2,72$ & $0,076^{* *}$ & $y=-0,177 x+138$ & 0,005 n.s. \\
\hline MEF50 (I/min) & $y=0,092 x-11,3$ & $0,20^{* *}$ & $y=0,279 x+51,7$ & 0,008 n.s. \\
\hline MEF25 (I/min) & $y=0,037 x-4,41$ & $0,11^{* *}$ & $y=0,092 x+74,3$ & 0,001 n.s. \\
\hline
\end{tabular}

Tab. 4 Größenabhängigkeit der Lungenfunktionsparameter lungengesunder männlicher Probanden $(\mathrm{N}=164)$.

n. s. nicht signifikant; ${ }^{*} \alpha<0,05 ;{ }^{* *} \alpha<0,01$; \# Lineare Regression der Ist-Werte angegeben als \%Soll der EGKS.

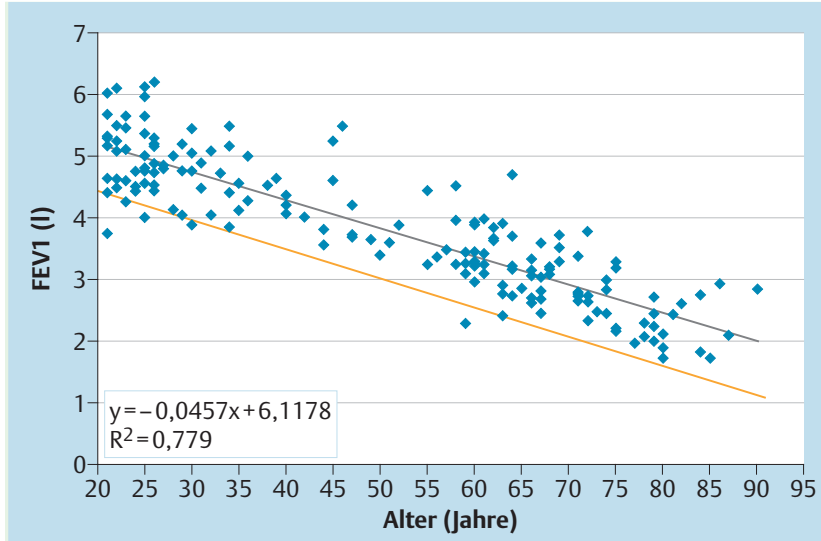

Abb. 1 Korrelation des $\mathrm{FEV}_{1}$ im Kontrollkollektiv mit dem Lebensalter von 164 lungengesunden Nichtrauchern im Alter zwischen 20 und 90 Jahren. Die rote Linie stellt den unteren Grenzwert dar (Sollwert - 1,64 × RSD).

\section{Korrelation der Lungenfunktionsparameter \\ mit dem Lebensalter}

Statische Lungenvolumina Die Altersabhängigkeit der Vitalkapazität $\left(\mathrm{VC}_{\mathrm{IN}}\right)$ der Kontrollprobanden kann mit einer linearen Funktion $\left(\mathrm{VC}_{\mathrm{IN}}=-0,046^{*}\right.$ Alter $\left.+7,01, \mathrm{r}=0,84\right)$ beschrieben werden. Für einen 80-jährigen Probanden beträgt $\mathrm{VC}_{\mathrm{IN}}$ im Mittel 3,30 L. Die $\mathrm{VC}_{\mathrm{IN}}$ lag bei den jüngeren Männern mit 5,73 $\pm 0,75 \mathrm{~L}$ bei $102 \pm 8,4 \%$ des Sollwertes und für die älteren Probanden bei $93,6 \pm 11,4 \%$. Die $\mathrm{VC}_{\mathrm{IN}}$ entsprach mit $101+13,1 \%$ bei den mittelalten Probanden dem Normwert. Bezogen auf die Referenzwerte der EGKS ergab sich eine signifikante Abnahme $(\alpha<0,01)$ der $\mathrm{VC}_{\text {IN }} \%$ Soll mit zunehmendem Lebensalter ( $\bullet$ Tab. 3).

Dynamische Lungenvolumina Die Altersabhängigkeit der Einsekundenkapazität $\left(\mathrm{FEV}_{1}\right)$ der lungengesunden Probanden kann ebenfalls mit einer linearen Funktion $\left(\mathrm{FEV}_{1}=-0,046 *\right.$ Alter $+6,11$; $r=0,88$ ) beschrieben werden. Für einen 80-jährigen Probanden beträgt die $\mathrm{FEV}_{1}$ im Mittel 2,70 $\pm 0,51 \mathrm{~L}$. Die $\mathrm{FEV}_{1}$ lag mit 4,83 $\pm 0,58 \mathrm{~L}$ bei den jüngeren Männern bei $108 \pm 9,9 \%$ des Sollwertes ( $\bullet$ Abb. 1), $105 \pm 13,7 \%$ für die mittelalten und für die älteren Probanden bei $97,1 \pm 12,4 \%$.

Überprüft man die Messwerte anhand der EGKS-Sollwerte, so liegen die Messwerte der jüngeren Probanden im Mittel signifikant $(\alpha<0,01)$ über der $100 \%$ Linie, die der mittelalten Probanden im Bereich der Sollwerte und die der älteren Probanden unterhalb der Sollwerte $(\bullet$ Abb. 2).

Die Altersabhängigkeit FVC der Kontrollprobanden kann mit einer linearen Funktion (FVC $=-0,047^{*}$ Alter $+7,03 ; r=0,74$ ) beschrieben werden. Für einen 80-jährigen Probanden beträgt die FVC im Mittel 3,54 $\pm 0,60$ L. Die FVC lag mit 5,69 $\pm 0,64 \mathrm{~L}$ bei den

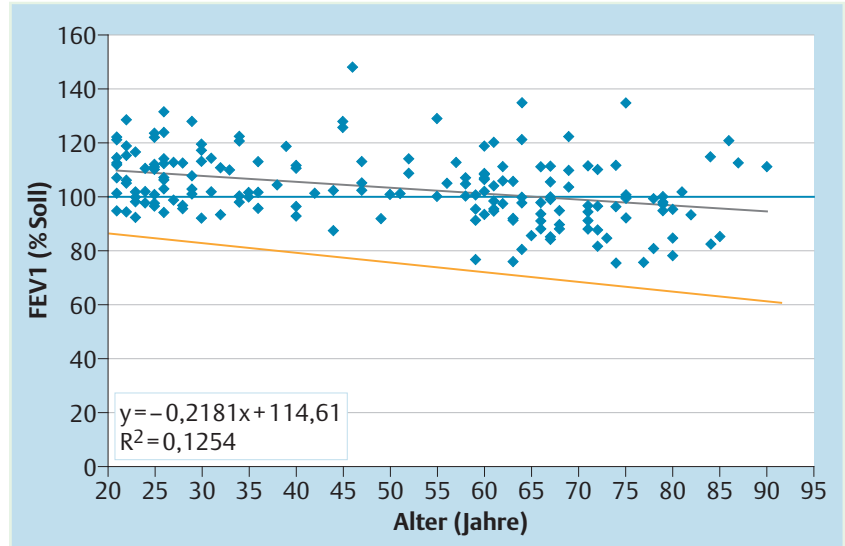

Abb. 2 Sollwertevergleich mit den Referenzwerten der EGKS für das $\mathrm{FEV}_{1}$ von lungengesunden nicht rauchenden Männern im Alter zwischen 20 und 90 Jahren. Die rote Linie stellt den unteren Grenzwert dar (Sollwert $-1,64 \times$ Soll).

jüngeren Männern bei $108 \pm 9,9 \%$ des Sollwertes und für die älteren Probanden bei $98,3 \pm 12,0 \%$.

Beim Vergleich mit den EGKS-Sollwerten liegen die Messwerte der jüngeren Probanden im Mittel signifikant $(\alpha<0,01)$ über der $100 \%$ Linie, die der mittelalten Probanden im Bereich der Sollwerte und die der älteren Probanden unterhalb der Sollwerte.

Die Altersabhängigkeit der relativen Einsekundenkapazität $\left(\mathrm{FEV}_{1} \% \mathrm{VC}\right)$ der Kontrollprobanden kann mit einer linearen Funktion $\left(\mathrm{FEV}_{1} \% \mathrm{VC}_{\mathrm{IN}}=-0,185 \mathrm{x}+90,0 ; \mathrm{r}=0,52\right)$ beschrieben werden. Für einen 80-jährigen Probanden beträgt die $\mathrm{FEV}_{1} \% \mathrm{VC}_{\mathrm{IN}}$ im Mittel $75,2 \%$. Die $\mathrm{FEV} 1 \% \mathrm{VC}_{\mathrm{IN}}$ lag bei den jüngeren Männern bei 102 $\pm 6,2 \%$ des Sollwertes und für die älteren Probanden bei 102 $\pm 8,6 \%$. Damit ist $\mathrm{FEV}_{1} / \mathrm{VC}_{\mathrm{IN}}$ über den gesamten Untersuchungsbereich im Mittel um 2\% größer als die EGKS-Referenzwerte.

Fluss-Volumen-Kurve Die Altersabhängigkeit des PEF der Kontrollprobanden kann mit einer linearen Funktion $(\mathrm{PEF}=-$ $0,078 \times$ Alter $+13,3 ; r=0,72$ ) beschrieben werden. Für einen 80 jährigen Probanden beträgt der PEF im Mittel 6,82 L/s. Der PEF lag bei den jüngeren Männern bei $112 \pm 13,8 \%$ des Sollwertes und für die älteren Probanden bei $96,7 \pm 15,9 \%$.

Die Altersabhängigkeit der maximalen exspiratorischen Stromstärke bei $50 \%$ der Vitalkapazität $\left(\mathrm{MEF}_{50}\right)$ der Kontrollprobanden kann mit einer linearen Funktion $\left(\mathrm{MEF}_{50}=-0,046 \times\right.$ Alter $+6,97$; $r=0,84$ ) beschrieben werden. Für einen 80 -jährigen Probanden beträgt die $\mathrm{MEF}_{50} \mathrm{im}$ Mittel 3,29 L/s. Die $\mathrm{MEF}_{50}$ lag bei den jüngeren Männern bei $101 \pm 20,1 \%$ des Sollwertes und für die älteren Probanden bei $95,0 \pm 24,9 \%$. 
Korrelation der Lungenfunktionsparameter

mit der Körpergröße

Statische Lungenvolumina Die Größenabhängigkeit der Vitalkapazität $\left(\mathrm{VC}_{\mathrm{IN}}\right)$ der Kontrollprobanden kann mit einer linearen Funktion $\left(\mathrm{VC}_{\mathrm{IN}}=0,104^{*}\right.$ Körpergöße $\left.-13,6, \mathrm{r}=0,68\right)$ beschrieben werden. Für einen $160 \mathrm{~cm}$ großen Probanden beträgt $\mathrm{VC}_{\mathrm{IN}}$ im Mittel 2,20 L, bei einem $190 \mathrm{~cm}$ großen Probanden bei 5,0 L. Bezogen auf die Referenzwerte der EGKS ergab sich eine geringe Zunahme der $\mathrm{VC}_{\mathrm{IN}}$ \%Soll mit zunehmender Körpergröße (৫ Tab.4).

Dynamische Lungenvolumina Die Größenabhängigkeit der Einsekundenkapazität $\left(\mathrm{FEV}_{1}\right)$ der Kontrollprobanden kann mit einer linearen Funktion $\left(\mathrm{FEV}_{1}=0,095 \times\right.$ Körpergröße $-12,9$; $\mathrm{r}=0,66$ ) beschrieben werden. Für einen $160 \mathrm{~cm}$ großen Probanden beträgt die $\mathrm{FEV}_{1}$ im Mittel 2,20 L, und für einen $190 \mathrm{~cm}$ großen Probanden 5,20 L ( $\bullet$ Abb. 3).

Überprüft man die Messwerte anhand der EGKS-Sollwerte, so liegen die Messwerte der kleineren Probanden im Mittel auf der $100 \%$ Linie und für die größeren Probanden leicht, aber nicht signifikant oberhalb der Sollwerte ( $\bullet$ Abb. 4).

\section{Diskussion}

Die verbreiteten Referenzwerte der EGKS für die ventilatorische Lungenfunktion können den heutigen Ansprüchen nicht mehr in allen Belangen genügen. Die Formeln sind retrospektiv aus verschiedenen Untersuchungen, die in den 70er-Jahren an unterschiedlichen Kollektiven durchgeführt wurden, kompiliert worden. Die Werte, die als Grundlage für die EGKS-Werte dienten, wurden an unterschiedlichen Probandenkollektiven erhoben. Seitdem haben sich die anthropometrischen Parameter erheblich geändert und die Messtechnik ist grundlegend verbessert worden. Durch die starre Definition des unteren Grenzwertes werden insbesondere ältere und kleinere Menschen in der Beurteilung einer Lungenfunktionseinschränkung erheblich benachteiligt. Die Definition ist nicht nur mitentscheidend für die Einstufung in gesund oder krank, sondern auch eine wesentliche Grundlage für gutachterliche Beurteilung.

Die Forderung nach einem kompletten Ersatz der Sollwerte der EGKS aufgrund der veränderten Bevölkerungsstrukturen ist nur unter großem finanziellen, materiellen und personellen Aufwand im Rahmen eines multizentrischen nationalen oder gar eines europäischen Forschungsvorhaben zu realisieren. In randomisierter Form müssten ca. 20000 Probanden aus den Melderegistern ermittelt werden, Raucher und erkrankte Personen ausgeschlossen und ausschließlich geeignete Probanden ausgewählt werden. Die Auswahl wird insbesondere in den höheren Altergruppen schwierig. Dies hat sich auch in unserer Untersuchung bestätigt. In einem umfassenden Referenzwerteprojekt sollten nicht nur die statischen und dynamischen Lungenvolumina, sondern auch die Parameter der Ganzkörperplethysmografie, die Diffusionseigenschaften der Lunge für $\mathrm{O}_{2}$ und $\mathrm{CO}_{2}$ und die Blutgase multizentrisch mit gleichen Mitteln untersucht werden. Eine solche Studie sollte bereits so angelegt werden, dass auch valide Aussagen über longitudinale Veränderungen gewonnen werden. Ein heute $190 \mathrm{~cm}$ großer 25-Jähriger wird mit 80 Jahren um einige cm kleiner als im jungen Erwachsenenalter sein. Querschnittsuntersuchungen, wie die der EGKS, beschreiben z. B. für die TLC keine Altersabhängigkeit. Nimmt denn die TLC im Laufe des Alterns gemeinsam mit der Körpergröße ab? Nur durch eine Längsschnittuntersuchung kann geklärt werden, ob sich signifikante Ände-

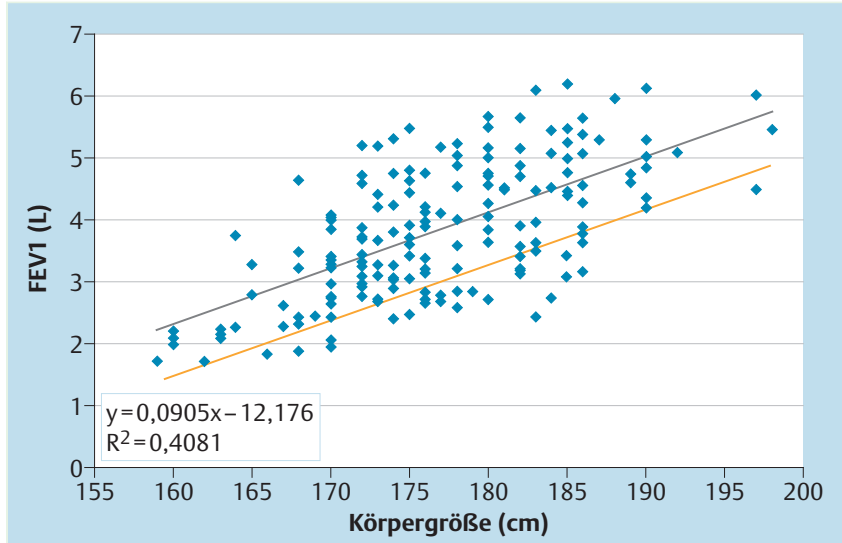

Abb. 3 Korrelation des $\mathrm{FEV}_{1}$ im Kontrollkollektiv mit der Körpergröße von 164 lungengesunden Nichtrauchern mit einer Körpergröße 159 bis $198 \mathrm{~cm}$. Die rote Linie stellt den unteren Grenzwert dar (Sollwert $-1,64 \times$ RSD).

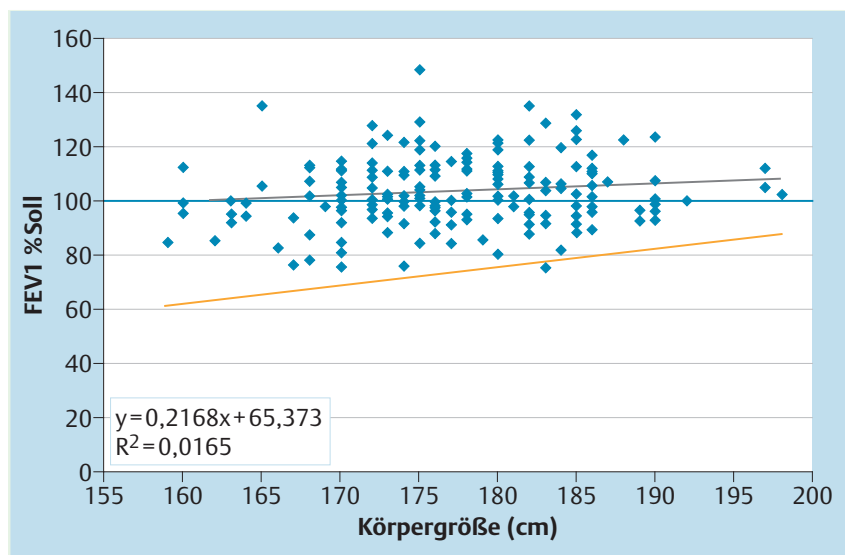

Abb.4 Sollwertevergleich mit den Referenzwerten der EGKS für das FEV von lungengesunden Nichtrauchern mit einer Körpergröße von 159 bis $198 \mathrm{~cm}$. Die rote Linie stellt den unteren Grenzwert dar (Sollwert $-1,64 \times$ Soll).

rungen der TLC in Korrelation zur Körpergröße nachweisen lassen.

\section{Vergleich der Referenzwertempfehlungen}

Die Veränderung in der Altersstruktur der Bevölkerung und die steigende Inzidenz chronisch obstruktiver Lungenerkrankungen bedingt, dass immer mehr ältere Personen einer Lungenfunktionsprüfung unterzogen werden müssen. Auch die Lebenserwartung von Patienten mit chronischen Lungenerkrankungen, wie z.B. die von silikosekranken Bergleuten, ist in den letzten Jahrzehnten erheblich gestiegen. So betrug nach Angaben der Bergbau-Berufsgenossenschaft im Jahre 2002 das mittlere Lebensalter von 15568 ehemaligen Bergleuten mit entschädigungspflichtiger Silikose (BK 4101) inzwischen 77,5 Jahre und liegt im Rahmen der übrigen männlichen Bevölkerung [20]. Nach Hochrechnungen der DE-STATIS werden wir bis zum Jahre 2040 einen Anstieg der über 65-Jährigen von derzeit 16,7 Millionen auf 23,5 Millionen Bundesbürger erreichen. Dabei wird der Anteil der mehr als 80-Jährigen größer als der der unter 20-Jährigen werden [21]. Ein heute 60-jähriger Mann hat nach Angaben des statistischen Bundesamtes noch eine mittlere Lebenserwartung von weiteren 19,7 Jahren. Die Einstufung der MdE beruht 
u.a. auf einer eingeschränkten Lungenfunktion. Die gebräuchlichen Referenzwerte sind jedoch in der Regel nur an Probanden bis zum 65. Lebensjahr erstellt worden. Gemeinsam ist den verschiedenen Empfehlungen die Alters- und Größenabhängigkeit der untersuchten Lungenfunktionsparameter, jedoch bestehen erhebliche Unterschiede in den Referenz- und Grenzwerten. Noch immer wird diskutiert, ob eine lineare Korrelation die Alters- und Größenabhängigkeit hinreichend beschreibt und ob weitere Parameter, wie z.B. die Rumpflänge, der Thoraxumfang oder der Body Mass Index einbezogen werden sollten. Für die computerisierten Messgeräte stellt die Verwendung von nichtlinearen Gleichungen für die Berechnung von Soll- und Sollgrenzwerten kein Problem dar.

\section{Ergebnisse des Referenzkollektivs}

Bei unserem Kollektiv lungengesunder männlicher Probanden konnte die Altersabhängigkeit über das 65. Lebensjahr hinaus bis zum 90. Lebensjahr für die meisten Parameter durchgehend mit einer linearen Funktion beschrieben werden. Exponentielle oder logarithmische Funktionen erbrachten keine relevanten Verbesserungen der Korrelation. Hinweise, dass die Lungenfunktion älterer „gesund gebliebener Probanden“ auf einem konstanten Wert bleiben oder im höheren Alter durch Summation von erkrankungsbedingten Veränderungen schneller abfallen, ließen sich, wie im Fletcher-Peto-Diagramm [22] dargestellt, an unserem kleinen - aber ausgesuchten - Probandengut nicht finden.

\section{Vergleich der EGKS zu den LuftiBus und SALPAPLDIA-Vorschlägen}

Die Sollwerte für die FEV 1 der EGKS und der LuftiBus -Studie unterscheiden sich nur im mittleren Altersbereich um bis zu 200 $\mathrm{mL}$. Für junge und ältere Probanden bestehen nur geringfügige Unterschiede ( $\bullet$ Abb. 5). Die Werte der SAPALDIA-Studie liegen für junge und mittelalte Pobanden um ca. $200 \mathrm{ml}$ höher und für mehr als 65-jährige etwa $300 \mathrm{ml}$. Die unteren Grenzwerte sind für die jüngeren der EGKS und der LuftiBus gleich, die der mittelalten und der älteren Probanden liegen etwa $200 \mathrm{ml}$ höher. Trotz

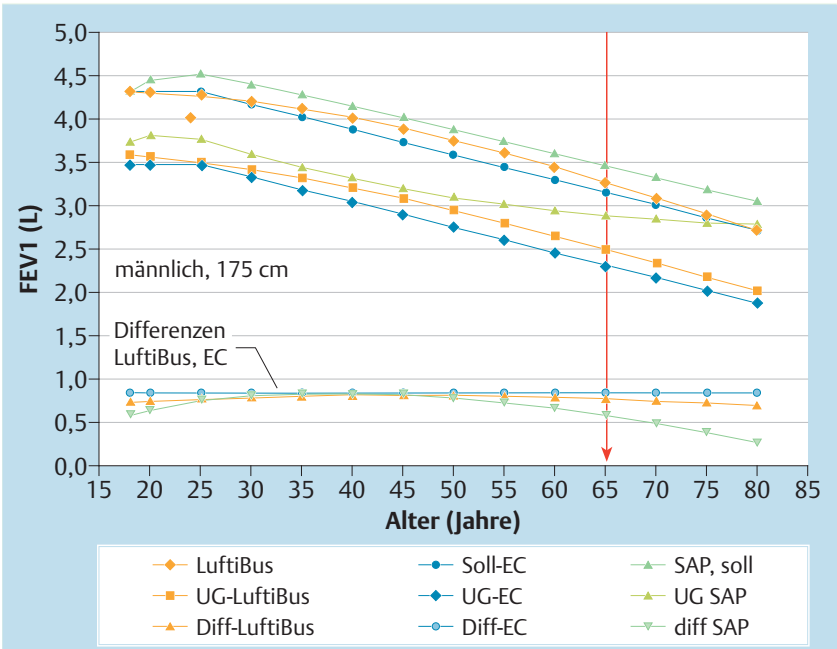

Abb.5 Vergleich der Sollwertkurven der $\mathrm{FEV}_{1}$ für $175 \mathrm{~cm}$ große Männer nach den Angaben der ECCS (blau), der LuftiBus-Studie (rot), und der SAPALDIA-Studie (grün). Beachte: die exponentiellen Kurven für die Sollwerte und die unteren Grenzwerte der LuftiBus-Studie sind nahezu identisch mit den Linearen Funktionen der ECCS, während sich die extrapolierten Sollwerte und unteren Grenzwerte der SAPALDIA- einander stark annähern. der altersbedingten Abnahme von mehr als 1,55 L zwischen den 25-Jährigen und den 80-Jährigen ist die Differenz des Sollwertes zur $5 \%$ Perzentile bei der EGKS und LuftiBus über den gesamten Altersbereich nahezu unverändert. In der ursprünglichen Version der SAPALDIA-Studie näherte sich der untere Grenzwert dem Referenzwert mit zunehmendem Alter an [6]. Aufgrund von neueren Modellannahmen, die die geringere Anzahl der älteren Probanden berücksichtigte, haben die Autoren die Formulierungen für den unteren Grenzwert neu definiert [23]. Damit verläuft auch in der SAPALDIA-Studie der untere Grenzwert nahezu parallel zum Referenzwert.

\section{Lungenfunktion beim älteren Menschen}

Die Thoraxwände werden mit zunehmendem Lebensalter steifer, d. h. die Compliance des Brustkorbs wird geringer, die Lunge wird jedoch leichter dehnbar und ihre elastischen Rückstellkräfte werden schwächer [24]. In der Folge nimmt das Residualvolumen (RV) zwischen dem 20. und 70. Lebensjahr um nahezu $50 \% \mathrm{zu}$. Gleichzeitig nimmt die Vitalkapazität in diesem Zeitraum um etwa $25 \%$ ab, da die totale Lungenkapazität (TLC) nahezu unverändert bleibt. Erhöhte Rückstellkräfte der Thoraxwände und die Elastizitätsabnahme der Lunge führen zur Verschiebung der Atemlage und der FRC in Richtung TLC. $\mathrm{FEV}_{1}$ und $\mathrm{VC}_{\mathrm{IN}}$ nehmen bis zum 20. Lebensjahr bei den Frauen bzw. bis zum 27. Lebensjahr bei den Männern zu, um dann im Laufe des Lebens kontinuierlich abzufallen $[25,26]$. Auf der Grundlage von Querschnittsund Längsschnittuntersuchungen wird eine schnellere Abnahme der $\mathrm{FEV}_{1}$ bei älteren Probanden ( $>65 \mathrm{Jahre}$ ) mit $38 \mathrm{~mL} / \mathrm{Jahr}$ diskutiert, im Vergleich dazu $20 \mathrm{~mL} / \mathrm{Jahr}$ bei jüngeren Probanden [27]. Eine schnellere Abnahme der $\mathrm{FEV}_{1}$ erfolgt bei Patienten mit bronchialer Hyperreagibilität [28]. Nur wenige Studien berichten von Lungenfunktionsuntersuchungen an einer größeren Anzahl von älteren Probanden. Ericsson [29] untersuchte 264 ältere Probanden, die jedoch jünger als 71 Jahre waren. Fowler [30] untersuchte 182 Londoner, die älter als 60 Jahre alt waren (44 älter als 75 Jahre und 23 älter als 80 Jahre). Enright [31] untersuchte 471 Probanden über 65 Jahre, von denen nur 10 Probanden über 80 Jahre alt waren.

Die beiden umfangreichsten Studien von Milne and Williamson [32] und DuWayne et al. [33] wurden bereits vor mehr als 25 Jahren publiziert und zeigen zum Teil widersprüchliche Ergebnisse. $\mathrm{FEV}_{1}$ und $\mathrm{FVC}$ nahmen mit zunehmendem Alter kontinuierlich $\mathrm{ab}$, während $\mathrm{FEV}_{1} \% \mathrm{VC}$ bei jüngeren Erwachsenen zunächst stabil waren und schließlich nach dem 55. bzw. 60. Lebensjahr bei Frauen und Männern abnahmen [33]. In der erstgenannten Untersuchung [32] beschrieben die Autoren jedoch eine Abnahme der $\mathrm{FEV}_{1} \% \mathrm{VC}$ nur bei den Frauen, während die Werte für Männer im Bereich von 60-90 Jahren konstant blieben.

Frühere Arbeiten zeigten, dass die Regressionsgleichungen, die auf Extrapolationen der Daten von jüngeren Probanden beruhten, die vorhergesagten Werte für $\mathrm{FEV}_{1}, \mathrm{FVC}$ und $\mathrm{FEV}_{1} / \% \mathrm{VC}$ für die älteren Probanden überschätzten. Enright et al. [31] verglichen die spirometrischen Messwerte älterer Probanden mit den nordamerikanischen Sollwerten [34] und fanden deutliche Unterschiede zu den Referenzwerten. Die Autoren berichteten von einer hohen Reproduzierbarkeit der Messergebnisse bei ca. 90\% der untersuchten Patienten [35].

Mit zunehmendem Lebensalter bekommt der lineare Verlauf der Fluss-Volumen-Kurve auch bei Probanden, die niemals in ihrem Leben geraucht haben, eine zunehmend konkave Form. Sie erinnert an Veränderungen in den peripheren Atemwegen [30]. Auch der PEF nimmt mit dem Lebensalter ab, wegen der großen Varia- 
bilität des PEF ist jedoch die Validität des Parameters gering [33]. Auch bei Lungengesunden wurde eine mittlere Variabilität des PEF von 8-10\% in einer Studie von 1223 Probanden im Altersbereich von 43 - 80 Jahren (MW 66 Jahre) gemessen.

Neben den altersbedingten Veränderungen der Compliance von Lunge und Thorax beeinflussen Veränderungen der Atemmuskelkraft wie auch die periphere Muskelstärke die statischen und dynamischen Lungenvolumina. Sowohl die maximalen inspiratorischen (MIP) als auch die maximalen exspiratorischen Drücke (MEP), gemessen bei RV oder FRC bzw. TLC, nehmen mit zunehmendem Lebensalter ab $[24,36]$. Die abnehmende Muskelkraft scheint insbesondere bei älteren Patienten relevant zu werden, wenn zusätzliche Atemmuskelarbeit bei akuten Pneumonien oder linksventrikulärem Herzversagen benötigt wird [28,37,38]. Mit steigendem Lebensalter wird die mitarbeitsabhängige Lungenfunktionsmessung häufig zum Problem. Bei ambulanten Patienten, die älter als 65 Jahre sind, ist eine Lungenfunktionsprüfung an etwa 90\% der Probanden durchführbar. Die Untersuchungsbedingungen bei stationären Patienten sind jedoch erschwert, da bei ihnen eine deutlich höhere Prävalenz von kognitiven Störungen besteht. Ganzkörperplethysmographische Untersuchungen sind in der Regel auch noch in höheren Altersgruppen möglich. In diesen Patientengruppen können auch Impedanzmessungen durchgeführt werden, die obstruktive Veränderungen ebenfalls quantifizieren [39].

\section{Individuelle Sollwerte}

Für einen 50-jährigen Mann von $175 \mathrm{~cm}$ Körpergröße beträgt die Differenz zwischen dem Sollwert des FEV 1 und den 5\% und 95\% Perzentilen ca. $\pm 25 \%$ vom Messwert. Ein Proband, der als Gesunder über ein $\mathrm{FEV}_{1}$ von $125 \%$ Soll, entsprechend der 95\% Perzentile, verfügt und aufgrund seiner beruflichen Exposition eine COPD erwirbt, muss ca. $50 \%$ seiner $\mathrm{FEV}_{1}$ einbüßen, um unter die $5 \%$ Perzentile zu fallen. Dies wäre vergleichbar dem funktionellen Verlust einer Lungenhälfte. Eine Möglichkeit, die Sensitivität der Sollwerte zu verbessern, wäre die Registrierung individueller Best-/Referenzwerte der/des jungen Erwachsenen, die dann im Laufe des Lebens als Bezugs- oder Sollwerte dienen. Auch die Deutsche Atemwegsliga unterstützt mit der Einführung des Lungenpasses die Gewinnung von Referenzwerten bei jungen Erwachsenen. Diese können in einem Diagramm, das die altersabhängigen Sollwerte für die $\mathrm{FEV}_{1}$ für die Körpergröße des Probanden darstellt, eingetragen werden. Trägt man die im Laufe des Lebens erhobenen Messwerte kontinuierlich in das Diagramm ein, so kann man ablesen, ob die Abnahme der $\mathrm{FEV}_{1}$ altersgemäß ist oder wie z. B. bei einer fortschreitenden Silikose eine schnellere Abnahme erfolgt.

\section{Zusammenfassung und Empfehlung für die Praxis und Klinik}

Der Altersgang von statischen und dynamischen Lungenfunktionsparametern geht in dem von uns untersuchten Kollektiv lungengesunder Probanden über den Gültigkeitsbereich der häufig verwendeten Sollwerte der EGKS $[4,16]$ unverändert weiter und kann für den Altersbereich von 20 bis 90 Jahren mit einem linearen Zusammenhang beschrieben werden. Die statischen und dynamischen Lungenvolumina jüngerer Probanden liegen im Mittel um wenige Prozente über den Sollwerten der EGKS, die Volumina der Probanden im mittleren Alter liegen nahe den Sollwerten und die der älteren Probanden im Mittel unter den EGKS-Sollwerten. Die Extrapolation der Lungenfunktionspara- meter über den Altersbereich der Sollwerte ist als Übergangslösung durchaus vertretbar.

Grenzwerte der SAPALDIA-Studie liegen zwar höher als die EGKS-Sollwerte, jedoch beschränkt sich der untersuchte Altersbereich lediglich zwischen 20 und 60 Jahren. Während der Abstand der 5\% Perzentilen in der ursprünglichen Publikation [6] mit zunehmendem Alter abnahm, was einer unserer Forderungen entspricht, wurde der untere Grenzwert nach engeren Kriterien neu definiert und ist nun wiederum nahezu konstant für ein unterschiedliches Lebensalter. Die Werte von Kuster [2] decken zwar einen Altersbereich von 18-80 Jahren ab, sind für junge und ältere Probanden nahezu identisch mit den EGKS-Werten und sind nur im mittleren Bereich um wenige $100 \mathrm{~mL}$ erhöht. Die Differenz zu den unteren Grenzwerten ist nahezu konstant. Die bisher weitläufig verwendeten Sollwerte der EGKS führen zu einer nicht vertretbaren Benachteiligung kleinerer und älterer Probanden und Patienten. Unter dem Aspekt der zunehmenden Altersstruktur und der angestiegenen Körpergröße sollte umgehend mit den Vorbereitungen für ein multizentrisches Forschungsprojekt zur Erhebung neuer Sollwerte für die Lungenfunktion begonnen werden.

\section{Literatur}

1 Hankinson JL, Odencrantz JR, Fedan KB. Spirometric reference values from a sample of the general U.S. population. Am J Respir Crit Care Med 1999; 159: 179-187

2 Kuster SP, Kuster D, Schindler $C$ et al. Reference equations for lung function screening of healthy never-smoking adults aged $18-80$ years. Eur Respir J 2008; 31: 860-868

3 Quanjer PH, Tammeling GJ, Cotes JE et al. Standardization of lung function Tests. Lung volumes and forced ventilatory flows. Eur Respir J 1993; 6 (Suppl 16): 5-40

4 Quanjer PH. Standardized lung function testing. Report Working Party Standardization of Lung Function Tests. European Community for Coal and Steel. Bull Eur Physiopathol Respir 1983; 19 (Suppl 5): 1-95

5 Criée CP, Berdel D, Heise D et al. Empfehlungen der Deutschen Atemwegsliga zur Spirometrie. Pneumologie 2006; 60: 576-584

6 Brändli $O$, Schindler $N$, Künzli $R$ et al. Lung function in healthy never smoking adults: reference values and lower limits of normal of a Swiss population. Thorax 1996; 51: 277-282

7 Coronell C, Orozco-Levi M, Mendez R et al. Relevance of assessing quadriceps endureance in patients with COPD. Eur Respir J 2004; 24: $129-136$

8 Miller MR, Hankinson JL, Brusasco V et al. Standardisation of Spirometry. Eur Respir J 2005; 26: 319-338

9 Baur X, Degens P, Heitmann R et al. Lung function testing: the dilemma of predicted values in relation to the individual variability. Respiration 1996; 63: $123-130$

10 Baur X, Isringhausen-Bley S, Degens $P$. Comparison of lung-function reference values. Int Arch Occup Environ Health 1999; 72: 69-83

11 Baur X, Degens P. Aktuelle Lungenfunktionswerte der mitteleuropäischen Bevölkerung (SALPALDIA-Studie). Atemw-Lungenkrkh 2004; 27: $32-41$

12 Degens $P$, Merget $R$. Reference values for spirometry of the European Coal and Steel Community: time to change. Eur Respir J 2008; 31: 687-688

13 Wise RA. The value of forced expiratory volume in 1 second decline in the assessment of chronic obstructive pulmonary disease progression. Am J Med 2006; 119 (Suppl 10): 4-11

14 Molfino NA. Genetic predisposition to accelerated decline of lung function in COPD. Int J Chron Obstruct Pulmon Dis 2007; 2: 117-119

15 Hnizdo E, Sircar K, Yan T et al. Limits of longitudinal decline for the interpretation of annual changes in $\mathrm{FEV}_{1}$ in individuals. Occup Environ Med 2007; 64: 701 - 707

16 Quanjer P, Tammeling GJ, Cotes JE et al. Lung volumes and forced ventilatory flows. Report of the working party standardization of lung functions tests. European Community for Steel and Coal. Eur Respir J 1993; 6 (Suppl 16): 5-40 
17 Marek W, Baur X. Ganzkörperplethysmographische Lungenfunktionsprüfungen: Messung von Atemwegswiderstand und intrathorakalem Gasvolumen, Sollwert/Messwertvergleich und Interpretation der Druck-Strömungs-Beziehung. Atemw-Lungenkrkh 1995; 21: 145-159

18 Marek W, Gespers H, Riebe J. Lungenfunktion und Blutgase lungegesunder älterer Probanden. Atemw-Lungenkrkh 2000; 26 (4): 189-199

19 Sachs L. Angewandte Statistik, Planung und Auswertung, Methoden und Modelle. Berlin, Heidelberg, New York: Springer, 1992

20 Bergbau-Berufsgenossenschaft. Jahresbericht 2002 der Bergbau-Berufsgenossenschaft. Hummitzsch W, Ulitzka N, eds 2003: 1-67

21 Birg H. Perspektiven der Bevölkerungsentwicklung in Deutschland und Europa - Konsequenzen für die sozialen Sicherungssysteme. Vortragsunterlagen für Sachverständigenanhörung des Bundesverfassungsgerichts in Karlsruhe. 2000: 1-33

22 Fletcher C, Peto R. The natural history of chronic airflow obstruction. B M J 1977; 1: 1645-1648

23 Brändli O, Leuenberger P, Schindler $N$ et al. Reestimated reference equations for the 5th percentiles of lung function variables in the adults population of Switzerland (SALPAIDIA-Study). Thorax 2000; 55: 173 174

24 Janssens PJ, Pache JC, Nicod LP. Physiological changes in respiratory function associated with ageing. Eur Respir J 1999; 13: 197-205

25 Knudson RJ, Slatin RC, Lebowitz MD et al. The maximal expiratory flowvolume curve: normal standards, variability, effects of age. Amer Rev Respir Dis 1976; 113: 587-599

26 Knudson RJ, Lebowitz MD, Holdberg CJ et al. Changes in maximal expiratory flow-volume curve with growth and aging. Am Rev Respir Dis 1983; 127: 725-734

27 Brandstetter RD, Kazemi H. Aging and the respiratory system. Med Clin N Am 1983; 67: 419-431
28 Crapo RO. The aging Lung. In: Mahler DA, ed. Pulmonary Disease in the Elderly Patient. New York: Marcel Dekker, 1993: 1-21

29 Ericsson P, Irnell L. Spirometric studies of ventilatory capacity in elderly people. Acta Med Scand 1969; 185: 179-184

30 Fowler RW, Pluck RA, Hetzel MR. Maximal expiratory flow-volume curves in Londoners aged 60 yrs and over. Thorax 1987; 42: 173-182

31 Enright PL, Kronmal RA, Higgins $M$ et al. Spirometry reference values for women and men 65 to 85 years of age. Am Rev Respir Dis 1993; 147: $125-133$

32 Amrein $R$, Keller R, Joos $H$ et al. Neue Normalwerte für die Lungenfunktionsprüfung mit der Ganzkörperplethysmographie. Dtsch Med Wochenschr 1969; 94: 1785-1793

33 DuWayne Schmidt C, Dickman ML et al. Spirometric standards for healthy elderly men and women: 532 subjects, ages 55 through 94 years. Am Rev Respir Dis 1973; 108: 933-939

34 Becklake M, Crapo RO, Buist B et al. Lung function testing: selection of reference values and interpretative strategies. Am Rev Respir Dis 1991; 144: $1202-1218$

35 Enright PL, Beck KC, Sherrill DL. Repeatability of spirometry in 18,000 adult patients. Am J Respir Crit Care Med 2004; 169: 235-238

36 Enright PL, Kronmal RA, Manolio TA et al. Respiratory muscle strength in the elderly relates and reference values. Am Rev Respir Dis 1994; 149: $430-438$

37 Evans $S$, Watson L, Hawkins $M$ et al. Respiratory muscle strength in chronic heart failure. Thorax 1995; 50: 625-628

38 Nishimura $Y$, Maeda H, Tanaka Ket al. Respiratory muscle strength and hemodynamics in chronic heart failure. Chest 1994; 105: 355 - 359

39 Carvalhaes-Neto N, Lorino H, Gallinari C et al. Cognitive assessment of lung function in the elderly. Am J Respir Crit Care Med 1995; 152 : $1611-1615$ 